\title{
HÁBITOS DE OCIO EN ESTUDIANTES DE MÚSICA: EL CASO DEL CONSERVATORIO DEL TOLIMA, COLOMBIA
}

\author{
HABITS DURING THE FREE TIME OF MUSIC STUDENTS: \\ CASE OF THE CONSERVATORY OF TOLIMA, COLOMBIA
}

\section{HÁBITOS DE LAZER DE ESTUDANTES DE MÚSICA: CASO DO CONSERVATÓRIO DE TOLIMA, COLÔMBIA}

\section{Hernán Gilberto Tovar Torres ${ }^{1}$}

1 Doctor en Ocio y Desarrollo Humano. Universidad de Deusto. Docente de Planta y Coordinador rea de Recreaci n y Ocio en la de la Universidad del Tolima. E-mail: hgtovar@ut.edu.co 


\section{RESUMEN}

Objetivo: identificar y describir los $\mathrm{h}$ bitos de ocio de los estudiantes de carreras de m sica del Conservatorio del Tolima, Colombia. Metodolog a: estudio descriptivo transversal de tipo cuantitativo. Hace parte de una investigaci $\mathrm{n} \mathrm{m} \mathrm{s}$ amplia sobre ocio y salud. Se analizan dos dimensiones del cuestionario elaborado para este fin, las caracter sticas de la formaci $n$ musical y los $h$ bitos de tiempo libre. Resultados: los datos revelan que el principal motivo por el que eligen los estudios de carreras de $\mathrm{m}$ sica es que se trata de una pasi $\mathrm{n}$ personal. Poca disponibilidad de tiempo libre en un $d$ a normal de estudio. Preferencias de ocio sedentarias y correspondidas con los estudios musicales, percibiendo alto grado de satisfacci n. Deseo de realizar principalmente actividades de ocio como viajar, visitar museos y actividades $f$ sico-deportivas, encontrando barreras como no tener tiempo y ausencia de oferta universitaria de ocio. Conclusi n: se identifica la necesidad de modificar $h$ bitos de ocio en este colectivo, con el fin de prevenir problemas de salud que aquejan al $\mathrm{m}$ sico durante su formaci $n$ universitaria.

PALABRAS CLAVE: tiempo libre, ocio, estudiantes de $\mathrm{m}$ sica.

\section{ABSTRACT}

Objective: To identify and describe the leisure habits of the music students of the Conservatorio del Tolima, Colombia. Methodology: Descriptive cross-sectional study of quantitative type. As part of a wider research on leisure and health, two dimensions of the ad hoc questionnaire, "the characteristics of musical training" and "habits of free time" are analyzed. Results: His data reveal that the main reason why they choose music studies is that it is a personal passion. Preferences of leisure sedentary and corresponded with the musical studies, perceiving high degree of satisfaction. They want to carry out mainly leisure activities such as traveling to visit museums and physical-sport activity, finding barriers such as not having time and lack of university offer. Conclusion: it is identified the need to modify leisure habits in this group to achieve greater prevention of health problems that afflict the musician during his university education.

KEYWORDS: free time, leisure, music students.

\section{RESUMO}

Objetivo: Identificar e descrever os $\mathrm{h}$ bitos de lazer de estudantes de $\mathrm{m}$ sica de Conservat rio de M sica de Tolima, Col mbia. Metodologia: Estudo quantitativo transversal. parte de uma pesquisa mais ampla sobre sa de e lazer, analisamos duas das dimens es do question rio ad hoc, "as caracte- 
r sticas da educaç o musical" e "h bitos de lazer". Resultados: os dados revelam que a principal raz o pela qual eles escolhem os estudos musicais que uma paix o pessoal. Prefer ncias sedent rias de lazer e combinados com estudos musicais, recebendo alto grau de satisfaç o. Eles baseiam-se principalmente atividades de lazer como viajar e visitar museus atividades $\mathrm{f}$ sicas e desportivas, encontrando barreiras como $\mathrm{n}$ o ter tempo e falta de oferta universidade. Conclus o: a necessidade de mudar os $\mathrm{h}$ bitos de lazer nesta comunidade para uma maior prevenç o de problemas de sa de que afligem o $\mathrm{m}$ sico durante a sua formaç o universit ria.

PALAVRAS-CHAVE: lazer, entretenimento, estudantes de m sica. 


\section{INTRODUCCIÓN}

El ocio, al que se refiere este estudio, es aquel que puede ser entendido como una vivencia cotidiana cultural, de pr cticas sociales m s bien I dicas de los sujetos (Gomes, 2015), y que adem s comprende requisitos relacionados con la significaci $n$, tales como la satisfacci n, el placer y el disfrute (Cuenca, 2004). Adem s, se le considera un derecho humano b sico al que todo el mundo debe tener acceso, independientemente de las condiciones individuales de raza, sexo, edad y estrato social, $\mathrm{m}$ s all de factores culturales, econ micos o laborales (World Leisure and Recreation Association, 1994).

En la actualidad, las actividades de ocio o de tiempo libre se armonizan muy bien con las m Itiples actividades de la vida cotidiana de los j venes, tales como las acad micas, laborales, familiares y de hogar, de ah que se consideren un elemento de fundamental valor en la vida de las personas (Fa lde \& Dap a, 2012). Esto se hace a $\mathrm{n} \mathrm{m}$ s evidente en el colectivo juvenil entre 15 y 29 a os, en el que se convierte en uno de los aspectos m s importantes (Funes, 2008), tal vez porque, a trav s de estas, esta poblaci $n$ encuentra una fuente para su bienestar y calidad de vida, dif cilmente accesible a trav $\mathrm{s}$ de otros mbitos de la vida (Trainor, Delfabbro, Anderson \& Winefield, 2010).

Con respecto a la etapa universitaria, el ocio expone algunas particulidades que lo diferencian del resto de actividades en la juventud. Esto se debe, b sicamente, a que las tareas universitarias y/o de estudio concentran toda su atenci n, relegando tareas juveniles como el trabajo dom stico, el uso y las actividades de tiempo libre, entre otras (Gil \& Men ndez, 1985). Respecto a las particularidades de las tareas de la vida universitaria expresa guila:

En el caso de los estudiantes universitarios, pasan por el tiempo de estudios, es decir, las exigencias espec ficas de la carrera que estu- 
dian, lo cual afecta las horas de clase y de estudio que requieren, las vinculaciones familiares o de pareja y la dedicaci $n$ complementaria a trabajos remunerados (2005, p.46).

Hablar concretamente del ocio en el colectivo juvenil universitario del siglo XXI, conlleva a condiciones de estudio y/o acad micas universitarias muy diferentes a las escolares por su mayor grado de responsabilidad. Esto quiz s tiene que ver con la reducci $\mathrm{n}$ del tiempo libre disponible en este colectivo, ante obligaciones como la asistencia a clases, la dedicaci n obligatoria a las tareas y las dem $\mathrm{s}$ actividades relacionadas con sus estudios, labores que segmentan el tiempo diario del universitario pr cticamente de manera generalizada (Comas, et al., 2003). De ah la importancia de aprovechar para sacar lo mejor de I, puesto que, de alguna manera, la Universidad representa cierta injerencia sobre el ocio, que, como se ha se alado, puede aportar beneficios en un proceso formativo y en el futuro profesional de una persona. Esto tambi $\mathrm{n}$ involucra todas aquellas actividades de ocio que el joven universitario puede realizar, tanto dentro como fuera de la universidad, en diferentes espacios, algo especialmente visible durante los fines de semana y en las vacaciones, por fuera de sus per odos de estudios.

Las ocupaciones de tiempo libre en los estudiantes de titulaci $\mathrm{n}$ universitaria de educaci $\mathrm{n}$ musical se combinan, sin duda, con una actividad laboral y/o estudio singular desarrollado pr cticamente a diario por este colectivo. Esto se refiere a que la actividad musical obliga al aprendiz a una dedicaci $\mathrm{n}$ prolongada durante largas horas de trabajo, sin movilizaci $\mathrm{n}$ de grandes masas musculares, siendo adem s una actividad caracterizada por el requerimiento de las mejores virtudes de la persona, esto es, las emocionales, las intelectuales, las psicol gicas y las $f$ sicas, de ah su consideraci $\mathrm{n}$ de alta exigencia corporal en los $\mathrm{m}$ sicos (Tubiana \& Amadio, 2000). De hecho, detr s de los resultados alcanzados por un $\mathrm{m}$ sico en lo acad mico y/o interpretativo, 
hay un proceso preparatorio de cientos de pr cticas instrumentales con posiciones y movimientos que no siempre son naturales, ensayos prolongados cargados de tensi $n$ y estr $s$, poco tiempo libre, e incluso horarios irregulares en comidas y horas de sue o.

En este escenario, ya sea durante su etapa como estudiante, o despu s como profesional, en los estudios realizados, el m si$\mathrm{co}$, con mucha frecuencia, puede presentar m Itiples molestias, alteraciones o problemas relacionados con su salud (Sard , 2003; Rosset \& Odam, 2010; Granada, 2011; Schoeb \& Zosso, 2012; Almonacid et al., 2013). En la actualidad, la literatura especializada indica que los $\mathrm{m}$ sicos sufren $\mathrm{m}$ Itiples problemas de salud, destac ndose los problemas m sculo-esquel ticos derivados de la interpretaci n musical (en adelante PME-I). Debido a ello, existe un amplio repertorio de investigaciones que demuestran, en $\mathrm{m}$ s de la mitad de los casos, la presencia de PME-I a lo largo de su carrera, en proporciones que oscilan entre el $30 \%$ y el $89 \%$, tanto en el caso de los $\mathrm{m}$ sicos profesionales, por la influencia de su actividad musical (Zaza, 1998; Guptill, 2010; Ackermann, Driscol \& Kenny, 2012), como en el caso de los m sicos en formaci n profesional y/o estudiantes (Via o, 2009; Ortiz, 2014).

Argumentos de peso que llevan a considerar, desde la perspectiva del investigador, la necesidad de identificar y describir lo que ocurre desde los $\mathrm{h}$ bitos durante el tiempo libre de este colectivo, con el fin de proporcionar $\mathrm{m} \mathrm{s}$ informaci $\mathrm{n}$ sobre un territorio desconocido al cual se le pretenden aportar nuevos conocimientos y pautas para la formulaci $\mathrm{n}$ de propuestas para el aporte a la salud del m sico, a partir de un espacio que complementa la vida cotidiana del estudiante de $\mathrm{m}$ sica, es decir, durante el tiempo libre.

\section{METODOLOGÍA}

Se realiz un dise o de investigaci n descriptivo, de tipo cuantitativo de corte transversal. La poblaci n o muestra de estudio se 
conform por 318 estudiantes de carreras de $\mathrm{m}$ sica, siendo el ciento por ciento (100\%), con un nivel de confianza del 95\%, y un margen de error del 5\%, lo que dio un total de 300 estudiantes en edades comprendidas entre 16 a 34 a os, del Conservatorio de m sica del Tolima, de la ciudad de Ibagu , Colombia.

El instrumento de recolecci $\mathrm{n}$ de datos construido expresamente para este fin (cuestionario ad hoc), fue validado a trav $\mathrm{s}$ de un juicio emitido por expertos (Cea, 2012) y un pre-test en una muestra piloto de 40 estudiantes. Las preguntas consideradas en las dos dimensiones analizadas por este estudio, obedecen a los trabajos de Garc a (2006, 2011), guila (2005), Ponce de Le n et al. (2010) y Romero \& Amador (2008), as como a una exhaustiva revisi $\mathrm{n}$ de la literatura especializada en $\mathrm{m}$ sicos.

Las variables de estas dimensiones incluyeron: caracter sticas sociodemogr ficas (g nero, edad, programa de estudio, semestre y especialidad musical); caracter sticas de la formaci $n$ musical (motivos elecci $\mathrm{n}$ de la carrera, horas de clase diarias en la universidad y horas adicionales dedicadas a estudiar y/o practicar $\mathrm{m}$ sica); $\mathrm{h}$ bitos de tiempo libre (horas tiempo libre disponible entre semana, actividades habituales de tiempo libre, nivel de satisfacci $n$, actividades deseadas y barreras para su realizaci $n$ ).

La administraci n del cuestionario a los participantes la realiz el investigador principal, autor del estudio, con apoyo de dos colaboradores formados previamente. Se efectu en las aulas, al comienzo de las clases, habi ndose solicitado previamente permiso a la rector a, seguido de una reuni $\mathrm{n}$ informativa con los formadores/docentes, en la cual se coordin los horarios de visita a la clase para la administraci $\mathrm{n}$ del instrumento.

El an lisis estad stico se realiz con el software SPSS versi n 21.0, que permiti un an lisis descriptivo univariado y bivariado de la informaci $n$, a partir de tablas de frecuencias $y$ porcentajes y el promedio y desviaci $n$ est ndar, sobre las caracter sticas de la poblaci $\mathrm{n}$ de inter $\mathrm{s}$ de este estudio. 


\section{RESULTADOS}

Despu s de analizar las 300 encuestas de los estudiantes, se destaca, en cuanto a las caracter sticas sociodemogr ficas, que el $69,3 \%$ son hombres. El $90 \%$ se encuentra entre los 16 y los 25 a os, siendo la edad promedio en hombres de 21,39 a os, con un $\mathrm{m}$ nimo de 16 y $\mathrm{m}$ ximo de 34; en mujeres, la edad promedio es de 20,27 a os, con un mimo de 16 y m ximo de 29 . El $73 \%$ estudia licenciatura en $\mathrm{m}$ sica. El $49 \%$ se encuentra entre el primer y segundo a o de estudio. El $42 \%$ son de la especialidad de cuerdas, siendo la que predomina sobre las dem s. En la tabla 1 se presenta el resto de las caracter sticas sociodemogr ficas de la muestra de estudio.

Tabla 1. Características sociodemográficas de los estudiantes de música

\begin{tabular}{lcc}
\hline \multicolumn{1}{l}{$n$} & $\%$ \\
\hline Sexo & & \\
\hline Hombre & 208 & $69,3 \%$ \\
Mujer & 92 & $30,7 \%$ \\
\hline Grupo de edad & & \\
\hline 16 - 20 años & 147 & $49,0 \%$ \\
21 - 25 años & 124 & $41,3 \%$ \\
26 - 30 años & 24 & $8,0 \%$ \\
31 y más años & 5 & $1,7 \%$ \\
\hline Programa de estudio & & \\
\hline Licenciatura en música & 221 & $73,7 \%$ \\
Maestro en música & 79 & $26,3 \%$ \\
\hline Semestre & & \\
\hline Un año (I y II) & 84 & $28,0 \%$ \\
Dos años (III y IV) & 64 & $21,3 \%$ \\
Tres años (V y VI) & 36 & $12,0 \%$ \\
Cuatro años (VII y VIII) & 50 & $16,7 \%$ \\
Cinco años o más (IX y X) & 66 & $22,0 \%$ \\
\hline
\end{tabular}




\begin{tabular}{lcc}
\hline & $n$ & $\%$ \\
\hline Especialidad musical & & \\
\hline Cuerdas & 125 & $42,2 \%$ \\
Canto & 71 & $23,4 \%$ \\
Vientos & 62 & $20,4 \%$ \\
Percusión & 38 & $12,7 \%$ \\
Director & 4 & $1,3 \%$ \\
\hline
\end{tabular}

Respecto al an lisis de la formaci n musical, se evidencia que la pasi $\mathrm{n}$ personal por sus estudios es el motivo predominante para elegir la carrera que estudian, en un $81 \%$. Asimismo, se observa que el promedio de horas diarias de clase que reciben los estudiantes es de 5 a 6 horas, en un 42\%; es decir, 4 de cada 10 estudiantes reciben en promedio entre 25 y treinta 30 horas semanales de clase. Asimismo, se puede ver que el $49 \%$ dedica de 2 a 4 horas diarias adicionales entre semana a la pr ctica de actividades relacionadas con los estudios musicales. No se encontraron diferencias porcentuales significativas entre stos y las variables sociodemogr ficas: g nero, grupo de edad, programa de estudio, semestre y especialidad musical, como se aprecia en la tabla 2 .

Tabla 2. Características de la formación musical de los estudiantes de música

\begin{tabular}{|c|c|c|c|c|c|c|}
\hline & \multirow[b]{3}{*}{$n$} & \multirow[b]{3}{*}{$\%$} & \multicolumn{4}{|c|}{ Programa de música } \\
\hline & & & \multicolumn{2}{|c|}{ Licenciatura } & \multicolumn{2}{|c|}{ Maestro } \\
\hline & & & $n$ & $\%$ & $n$ & $\%$ \\
\hline \multicolumn{7}{|l|}{ Motivos elección carrera } \\
\hline Es una pasión & 244 & $81,1 \%$ & - & - & - & - \\
\hline Es fácil encontrar trabajo & 33 & $11,0 \%$ & - & - & - & - \\
\hline No acceder a otras & 20 & $6,7 \%$ & - & - & - & - \\
\hline Impedimento económico & 2 & $0,9 \%$ & - & - & - & - \\
\hline Carrera fácil & 1 & $0,3 \%$ & - & - & - & - \\
\hline
\end{tabular}




\begin{tabular}{|c|c|c|c|c|c|c|}
\hline & & & \multicolumn{4}{|c|}{ Programa de música } \\
\hline & \multirow[b]{2}{*}{$n$} & \multirow[b]{2}{*}{$\%$} & \multicolumn{2}{|c|}{ Licenciatura } & \multicolumn{2}{|c|}{ Maestro } \\
\hline & & & $n$ & $\%$ & $n$ & $\%$ \\
\hline \multicolumn{7}{|c|}{ Horas asistencia diaria clase } \\
\hline Más de 6 horas & 37 & $12,7 \%$ & - & $8,1 \%$ & - & $10,5 \%$ \\
\hline De 5 a 6 horas & 126 & $42,0 \%$ & - & $64,4 \%$ & - & $61,6 \%$ \\
\hline De 2 a 4 horas & 115 & $38,6 \%$ & - & $24,7 \%$ & - & $23,3 \%$ \\
\hline Menos de 2 horas & 20 & $6,7 \%$ & - & $2,8 \%$ & - & $4,6 \%$ \\
\hline \multicolumn{7}{|c|}{$\begin{array}{l}\text { Horas adicionales dedicadas } \\
\text { a estudio y/o practica } \\
\text { música }\end{array}$} \\
\hline 5 horas y más diarias & 36 & $12,4 \%$ & - & $20,5 \%$ & - & $6,8 \%$ \\
\hline De 2 a 4 horas & 142 & $48,7 \%$ & - & $57,7 \%$ & - & $37,5 \%$ \\
\hline Menos de 2 horas & 109 & $34,4 \%$ & - & $20,3 \%$ & - & $52,3 \%$ \\
\hline Nada o casi nada & 13 & $4,5 \%$ & - & $1,5 \%$ & - & $3,4 \%$ \\
\hline
\end{tabular}

Respecto a los $\mathrm{h}$ bitos de ocio en el tiempo libre, se observa una tendencia hacia la percepci $\mathrm{n}$ de poco tiempo libre disponible en este colectivo, por cuanto se obtuvo un mayor porcentaje de $44,7 \%$, que dispone de menos y hasta 3 horas en un d a normal de estudio. Las actividades $\mathrm{m} \mathrm{s}$ habituales de tiempo libre son sedentarias, y se relacionan concretamente con los estudios musicales, esto es, escuchar $\mathrm{m}$ sica $(91 \%)$, y tocar instrumentos musicales (90\%); sin embargo, excepto estas, las dem s actividades habituales de los estudiantes de $\mathrm{m}$ sica muestran porcentajes distintos en funci n del sexo; por ejemplo, los hombres se inclinan $\mathrm{m}$ s hacia ver deporte espect culo (24\%), hacer actividades $\mathrm{f}$ sico-deportivas (54\%), estudiar $\mathrm{m} \mathrm{s}$ tiempo (56\%) y jugar juegos de mesa/sal n (18\%). Mientras a las mujeres les interesa m s estar en familia $(80 \%)$, ir de compras $(20 \%)$, ir a cine $(44 \%)$, ver pel culas $(74 \%)$ y estar en pareja (53\%).

Asimismo, se manifiesta en este colectivo un alto grado de satisfacci $n$ con las actividades de tiempo libre que realiza 
$(72,7 \%)$, y se detecta, entre las actividades que ahora no hacen y desear an realizar, un inter $\mathrm{s}$ hacia viajar y hacer fotografa $(40,7 \%)$, visitar museos $(39,1 \%)$ y realizar pr cticas $\mathrm{f}$ sicodeportivas $(36,7 \%)$. Finalmente, entre las principales barreras percibidas por los estudiantes para las actividades de tiempo libre deseadas, se encuentran la falta de tiempo (57,9\%), la falta de oferta universitaria $(55,8 \%)$ y la carencia de instalaciones (50,4\%). En la tabla 3 se observa el resto de los resultados relacionados con los $\mathrm{h}$ bitos de tiempo libre de este colectivo.

Tabla 3. Hábitos de ocio en el tiempo libre de los estudiantes de música

\begin{tabular}{|c|c|c|c|c|c|c|}
\hline & \multicolumn{6}{|c|}{ sexo } \\
\hline & \multirow[b]{2}{*}{$n$} & \multirow[b]{2}{*}{$\%$} & \multicolumn{2}{|c|}{ Hombre } & \multicolumn{2}{|c|}{ mujer } \\
\hline & & & $n$ & $\%$ & $n$ & $\%$ \\
\hline \multicolumn{7}{|l|}{$\begin{array}{l}\text { Horas de tiempo libre } \\
\text { disponible }\end{array}$} \\
\hline Menos y hasta 3 horas & 134 & $44,7 \%$ & - & $45,7 \%$ & - & $42,4 \%$ \\
\hline De 4 a 5 horas & 116 & $38,7 \%$ & 76 & $36,5 \%$ & 40 & $43,5 \%$ \\
\hline De 6 a 7 horas & 37 & $12,3 \%$ & - & $13,5 \%$ & - & $9,8 \%$ \\
\hline Más de 7 horas & 13 & $4,3 \%$ & - & $3,6 \%$ & - & $4,3 \%$ \\
\hline \multicolumn{7}{|l|}{$\begin{array}{l}\text { Actividades habituales de } \\
\text { tiempo libre }\end{array}$} \\
\hline Escuchar música & & $91,0 \%$ & - & $92 \%$ & - & $89 \%$ \\
\hline Tocar instrumentos musicales & & $90,3 \%$ & - & $91 \%$ & - & $88 \%$ \\
\hline Estar con la familia & & $70,3 \%$ & - & $66 \%$ & - & $80 \%$ \\
\hline $\begin{array}{l}\text { Ver televisión o películas de } \\
\text { video }\end{array}$ & & $59,3 \%$ & - & $54 \%$ & - & $74 \%$ \\
\hline $\begin{array}{l}\text { Salir con amigos o estar en } \\
\text { tienda o bar }\end{array}$ & & $57,0 \%$ & - & - & - & - \\
\hline Estudiar más tiempo & & $52,8 \%$ & - & $56 \%$ & - & $47 \%$ \\
\hline $\begin{array}{l}\text { Practicar actividades } \\
\text { físico-deportivas }\end{array}$ & & $50,5 \%$ & - & $54 \%$ & - & $40 \%$ \\
\hline Leer libros, revistas & & $50,0 \%$ & - & - & - & - \\
\hline
\end{tabular}




\begin{tabular}{|c|c|c|c|c|c|c|}
\hline & \multirow[b]{3}{*}{$n$} & \multirow[b]{3}{*}{$\%$} & \multicolumn{4}{|c|}{ sexo } \\
\hline & & & \multicolumn{2}{|c|}{ Hombre } & \multicolumn{2}{|c|}{ mujer } \\
\hline & & & $n$ & $\%$ & $n$ & $\%$ \\
\hline \multicolumn{7}{|l|}{$\begin{array}{l}\text { Grado de satisfacción con } \\
\text { actividades }\end{array}$} \\
\hline Satisfecho y muy satisfecho & 218 & $72,7 \%$ & - & - & - & - \\
\hline No estoy seguro & 59 & $19,7 \%$ & - & - & - & - \\
\hline $\begin{array}{l}\text { Insatisfecho y muy } \\
\text { insatisfecho }\end{array}$ & 23 & $7,6 \%$ & - & - & - & - \\
\hline \multicolumn{7}{|l|}{$\begin{array}{l}\text { Actividades tiempo libre } \\
\text { deseadas de realizar }\end{array}$} \\
\hline Viajar y hacer fotografía & & $40,7 \%$ & - & - & - & - \\
\hline Visitar museos & & $39,1 \%$ & - & - & - & - \\
\hline $\begin{array}{l}\text { Practicar actividades } \\
\text { físico-deportivas }\end{array}$ & & $36,7 \%$ & - & - & - & - \\
\hline Realizar cursos culturales & & $35,5 \%$ & - & - & - & - \\
\hline $\begin{array}{l}\text { Leer libros, revistas } \\
\text { (partituras, dibujar) }\end{array}$ & & $29,3 \%$ & - & - & - & - \\
\hline $\begin{array}{l}\text { Ir a cine, teatro, espectáculos } \\
\text { artísticos }\end{array}$ & & $25,3 \%$ & - & - & - & - \\
\hline Hacer trabajos manuales & & $20,7 \%$ & - & - & - & - \\
\hline Juegos de mesa y salón & & $16,5 \%$ & - & - & - & - \\
\hline \multicolumn{7}{|l|}{$\begin{array}{l}\text { Barreras de no realización de } \\
\text { actividades }\end{array}$} \\
\hline Por miedo a lesionarme & 28 & $10,0 \%$ & & & & \\
\hline Mis amistades no hacen & 39 & $18,4 \%$ & & & & \\
\hline Otras & 79 & $26,4 \%$ & & & & \\
\hline Carencia de instalaciones & 150 & $50,4 \%$ & & & & \\
\hline No hay oferta universitaria & 165 & $55,8 \%$ & & & & \\
\hline Por no tener tiempo & 172 & $57,9 \%$ & & & & \\
\hline
\end{tabular}




\section{DISCUSIÓN}

El presente estudio describe los $\mathrm{h}$ bitos durante el tiempo libre de estudiantes de $\mathrm{m}$ sica. Su importancia gira en torno a que la identificaci $\mathrm{n}$ y conocimiento del marco complementario de los estudios universitarios de $\mathrm{m}$ sica pueden ejercer un importante papel en la calidad de vida presente y futura de este colectivo, constituyendo un punto de partida importante para orientar acciones concretas hacia el desarrollo humano, el bienestar y la prevenci $\mathrm{n}$ de los problemas generados por los excesos de la pr ctica musical, por lo que resulta clave actuar desde la base de la formaci $\mathrm{n}$ profesional de $\mathrm{m}$ sica.

Para describir las caracter sticas sociodemogr ficas de estudio musical y de tiempo libre, se elaboraron preguntas cerradas, ya acotadas en el cuestionario (precodificadas). Esta elecci $n$ tiene como ventaja la facilidad de estandarizaci $\mathrm{n}$ y adem $\mathrm{s}$ brinda mayor facilidad de respuesta en el colectivo en el que se realiz el estudio. Adem s, porque la informaci $n$ que se ha de proporcionar es muy personal, por lo que a veces es dif cil expresarla verbalmente.

Los hallazgos del presente estudio se alaron, en primer lugar, el hecho de que los estudios musicales se deben a una pasi $n$ personal de los estudiantes de $\mathrm{m}$ sica, datos que no pueden ser ampliamente discutidos al no encontrar investigaciones que lo permitan. Sin embargo, s se logr interpretar c mo la condici $n$ de pasi $\mathrm{n}$ encontrada, ayuda a entender por qu la alta dedicaci $\mathrm{n}$ de tiempo diario de estudio musical (entre tiempo obligatorio de clases y tiempo adicional), as como la poca disponibilidad de tiempo libre encontrada en un d a normal de estudios en este colectivo, que result incluso $\mathrm{m} \mathrm{s}$ reducido con respecto a universitarios iberoamericanos de otras carreras (Barrionuevo, 2012; OTIUM, 2011; Castillo \& Gim nez, 2011; Cuenca \& Nieto, 2010; guila, 2005). 
Los datos analizados sobre las actividades habituales de tiempo libre realizadas, nos permiten inferir un aumento significativo de riesgos en salud de este colectivo a trav $\mathrm{s}$ los $\mathrm{h}$ bitos de tiempo libre, principalmente por el predominio de actividades similares a sus estudios de carreras de m sica en 9 de cada 10 estudiantes, como tocar instrumentos musicales y escuchar $\mathrm{m}$ sica, as como por la ausencia de pr cticas $\mathrm{f}$ sico-deportivas en 1 de cada 2 de estos.

Dado que el alto grado de sedentarismo se denota como una coincidencia en los j venes una vez inician la vida universitaria, como lo confirma una recopilaci $n$ de estudios iberoamericanos (Bast as \& Stiepovich, 2014), e incluso de investigaciones en m sicos (Via o, 2009; Betancor, 2011), tales hallazgos ponen en evidencia la necesidad de plantear estrategias diferentes de ocupaci $\mathrm{n}$ de tiempo libre de este colectivo, tanto en la universidad como fuera de ella. Asimismo, el sexo, en este estudio, se mostr como una variable de participaci n o distanciamiento de la actividad $f$ sica y el deporte, coincidiendo con los resultados obtenidos en otros estudios, en este colectivo (Romero \& Amador, 2008; Pav n \& Moreno, 2008; D az, Gonz lez \& Arrieta, 2014; Torres, 2015).

A pesar de mostrar marcadas diferencias con las actividades habituales de ocio en el colectivo de estudiantes de $\mathrm{m}$ sica, como tocar instrumentos y escuchar $\mathrm{m}$ sica, frente a ver televisi $\mathrm{n}$ y usar el ordenador, $\mathrm{m}$ viles del resto de universitarios iberoamericanos (Ponce Le $\mathrm{n}$ et al., 2010; Ochoa \& Rangel, 2012; D az, Gonz lez \& Arrieta, 2014), los hallazgos del presente estudio coinciden en los niveles de satisfacci $n$ obtenidos con las ocupaciones de tiempo libre en otras investigaciones realizadas en poblaci $\mathrm{n}$ universitaria iberoamericana (OTIUM, 2011; Ponce de Le $\mathrm{n}$ et al., 2010; $M$ ndez, 2008; guila, 2005), quedando claro que las actividades de ocio y tiempo libre de los j venes universitarios, incluidos los $\mathrm{m}$ sicos, parecen moverse a partir de una b squeda alta de satisfacci n con lo que hacen. 
Respecto a los principales hallazgos alcanzados en este estudio sobre las actividades que se desean realizar, y en el momento no hacen parte de su tiempo libre (viajar, visitar museos y practicar actividad f sico-deportiva), se observan coincidencias con los resultados obtenidos en otros estudios con universitarios, en los que se desea ser $\mathrm{f}$ sicamente $\mathrm{m}$ s activos ( guila, 2005), y asimismo en poblaci $\mathrm{n}$ joven en general (Garc a, 2011). Sin embargo, la principal inferencia en este estudio tiene que ver con la importancia de querer realizar actividades $\mathrm{f}$ sicodeportivas por encima de actividades $\mathrm{m}$ s sedentarias como ir a cine, ver televisi $n$, navegar en internet o jugar videojuegos, como ocurre con este colectivo. Adem s, porque el deseo y la motivaci $n$ es un elemento de enganche y continuidad de estas actividades que pueden aportar a las necesidades de singularidad del $\mathrm{m}$ sico en proceso de formaci $\mathrm{n}$ profesional.

Carecer de tiempo se identifica como la principal barrera para las actividades de tiempo libre en este colectivo, siendo un dato que coincide ampliamente con los resultados obtenidos en diversos estudios en universitarios Iberoamericanos (OTIUM, 2011), con consideraciones como adecuar la oferta de actividad f sica y aumentar los h bitos del alumnado universitario (Castillo \& Gim nez, 2011).

\section{CONCLUSIONES}

Los $\mathrm{h}$ bitos de tiempo libre en los estudiantes de $\mathrm{m}$ sica descritos en esta investigaci $n$, evidencian un alto grado de pasi $n$ personal por los estudios en este colectivo, lo cual ha llevado a una mayor dedicaci $n$ a sus estudios y a una disminuci $n$ del tiempo libre, especialmente entre semana. Tambi n se encontr que las actividades predominantes de tiempo libre son una prolongaci $\mathrm{n}$ de las actividades de estudio del $\mathrm{m}$ sico, entre las que sobresalen tocar instrumentos y escuchar $\mathrm{m}$ sica, respecto a lo 
cual los estudiantes se encuentran altamente satisfechos con la forma en que ocupan su tiempo libre. Asimismo, se observ , con excepci $\mathrm{n}$ de las actividades relacionadas con la $\mathrm{m}$ sica, como el sexo es uno de los aspectos de influencia sobre la participaci n o distanciamiento de ciertas actividades de tiempo libre. Sin embargo, en los estudiantes existe una semejanza en cuanto a pasar un tiempo libre de manera m s sedentaria, aunque es a $\mathrm{n} \mathrm{m} \mathrm{s}$ notorio en las mujeres. En este mismo sentido, las dem $\mathrm{s}$ dimensiones estudiadas identificaron un alto inter $\mathrm{s}$ por realizar actividades de tiempo libre $\mathrm{m} \mathrm{s}$ activas, tales como viajar, visitar museos y actividades $f$ sico-deportivas, presentando adem s como principales barreras la falta de tiempo libre disponible, la no oferta universitaria y la carencia de instalaciones de manera generalizada. As, se deja ver que existe una clara concepci n por participar en otro tipo de actividades diferentes a las habituales, que podr an beneficiar su salud y complementar sus estudios.

Con las conclusiones planteadas sobre los actuales $\mathrm{h}$ bitos durante el tiempo libre, que mostraron tendencias sedentarias y actividades repetitivas de la $\mathrm{m}$ sica en los estudiantes, se puede afirmar que, a trav s del ocio y/o tiempo libre, aumentan las posibilidades de estar en situaciones de riesgo de salud, dadas las condiciones de singularidad identificadas por la literatura especializada, con alta exposici n a factores de riesgo asociados a su campo de profesional, a que se enfrenta a diario el colectivo de $\mathrm{m}$ sicos estudiantes. Tambi $\mathrm{n}$, dejan ver un escenario de revalorizaci n positiva por parte de los estudiantes hacia el tiempo libre, al identificar un punto de partida para una oferta de $h$ bitos f sicamente $\mathrm{m} s$ activos en la universidad, que sea acorde con las necesidades del $\mathrm{m}$ sico en formaci $\mathrm{n}$, con lo cual se recoge adem s lo atinente con las barreras detectadas, como escasez de tiempo libre, ausencia de ofertas especializadas y falta de instalaciones para este colectivo, en este estudio. 


\section{REFERENCIAS}

1. Ackermann, B., Driscol, T., \& Kenny, D. (2012). Musculoskeletal Pain and Injury in Professional Orchestral Musicians in Australia. Medical Problems of Performing Artists, 27(4),181-187.

2. guila, S. (2005). Ocio, j venes y postmodernidad. Almer a, Espa a: Servicio de Publicaciones Universidad de Almer a.

3. Alberto, S., Hernando, J., \& Fern ndez, A. (1996). Gesti $n$ y direcci $n$ de las empresas deportivas. Teor a y Pr ctica. Madrid: Gymnos.

4. Almonacid, G., Gil, I., L pez, I., \& Bolace, I. (2013). Trastornos m sculoesquel ticos en $\mathrm{m}$ sicos profesionales: revisi $\mathrm{n}$ bibliogr fica. Revista de Medicina y Seguridad del trabajo, 59 (230) 124-145.

5. OTIUM Asociaci n Iberoamericana de Estudios de Ocio (2011). Estudio de la experiencia de ocio en Iberoam rica en j venes universitarios. Espa a: OTIUM.

6. Barrionuevo, D. (2012). Diagn stico de la utilizaci n del tiempo libre de los estudiantes de las ingenier as de la modalidad presencial de la Escuela Polit cnica del Ej rcito. Revista Latinoamericana de Recreaci $n, 1(2), 1-12$.

7. Bast as, E., \& Stiepovich, B. (2014). Una revisi n de los estilos de vida de estudiantes universitarios iberoamericanos. Ciencia y Enfermer a, 20(2), 93-101.

8. Betancor, A. (2011). $\mathrm{H}$ bitos de actividad $\mathrm{f}$ sica en $\mathrm{m}$ sicos de orquestas sinf nicas profesionales: un analisis emp rico de mbito internacional (Tesis Doctoral). Espa a: Universidad de las Palmas de Gran Canaria.

9. C rdenas, S., Mart nez, F., \& Vergara, K. (2014). Niveles de actividad $\mathrm{f}$ sica asociados a factores sociodemogr ficos, antropom tricos y conductuales en universitarios de Cartagena. Salud Uninorte, 30(3).

10. Castillo, E., \& Gim nez, F. (2011). Practice of physical activity of students of the University of Huelva. International Journal of Medicine and Science of Physical Activity and Sport, 11(41), 127-144.

11. Cea, M. (2012). Fundamentos y aplicaciones en metodolog a cuantitativa. Madrid: $\mathrm{S}$ ntesis.

12. Comas, D., Aguinaga, J., Andr s, F., Espinosa, A., \& Ochaita, E. (2003). $\mathrm{J}$ venes y estilo de vida. Valores y riesgos de los $\mathrm{j}$ venes urbanos. Madrid: INJUVE.

13. Cuenca, M. (2004). Pedagog a del ocio: modelos y propuestas. Bilbao: Publicaciones Universidad de Deusto.

14. Cuenca, M., \& Nieto O. (2014). Valor del ocio en un grupo de universitarios de pregrado de la Universidad de Caldas (Colombia). Recu- 
perado desde: http://es.slideshare.net/ociogune/valor-del-ocio-en-ungrupo-de-universitarios-de-pregrado

15. D az, S., \& Gonz lez, F., \& Arrieta, K. (2014). Niveles de actividad $\mathrm{f}$ sica asociados a factores sociodemograficos, antropometricos y conductuales en universitarios de Cartagena (Colombia). Salud Uninorte, 30(3), 405-417.

16. Fa Ide, J., \& Dap a, M. (2012). Las evidencias cient ficas en el mbito del ocio y del tiempo libre. Programas eficaces. En: M. Isorna \& D. Saavedra (comps.), Prevenci $\mathrm{n}$ de drogodependencias y otras conductas adictivas (pp.367-375). Madrid, Espa a: Pir mide.

17. Funes, M. (2008). Informe Juventud en Espa a 2008. Recuperado desde: http://www.injuve.es/observatorio/demografia-e-informaci n-ge neral/informe-juventud-en-espa a-2008

18. Garc a, M. (2006). Postmodernidad y deporte: entre la individualizaci $n$ y la masificaci n. Encuesta sobre h bitos de los espa oles 2005. Madrid: Ministerio de Educaci n, Cultura y Deporte. Consejo Superior de Deportes.

19. Garc a, M. (2011). Ideal democr tico y bienestar personal. Encuesta sobre los h bitos deportivos de Espa a 2010. Madrid: Consejo Superior de Deportes.

20. Gil, E., \& Men ndez, E. (1985). Ocio y pr cticas culturales de los j venes. Madrid: INJUVE.

21. Gomes, C. (2015). Lazer necessidade humana e dimensao da cultura. Revista Brasileira de Estudos do Lazer, 1(1), 3-20.

22. Granada, J. (2011). An lisis de las patolog as musculo-esquel ticas en los clarinetistas. Revista Complutense de Investigaci n en Educaci n Musical, 8(3), 1-17.

23. Guptill, C. (2010). Survivors on the edge: The lived experience of professional musicians with playing-related injuries. Canad : Western University.

24. M ndez, R. (2008). Los j venes universitarios y su (des)orientaci $n$ ante los nuevos retos que plantea el EEES. Educatio Siglo XXI, 26, 197-224.

25. Ochoa, M., \& Rangel, L. (2012). El tiempo libre de los estudiantes universitarios de la Facultad de Comercio y Administraci n de Tampico. CienciaUAT, 6(3), 20-26.

26. Ortiz, M. (2014). Prevalencia de s ntomas osteomusculares en miembros superiores y los probables factores de riesgo asociados en los estudiantes de $\mathrm{m}$ sica en una instituci $\mathrm{n}$ universitaria de Bogot , Colombia (Tesis de maestr a). Bogot , Colombia: Universidad del Rosario, Maestr a en Salud Ocupacional y Ambiental.

27. Pav n, A., \& Moreno, J. (2008). Actitud de los universitarios ante la pr ctica f sico-deportiva: diferencias por g neros. Revista de Psicolog a del Deporte, 17(1), 7-23. 
28. Ponce de Le n, A., Sanz, E., Ramos, R., \& Valdemoros, M. (2010). MACOFYD Cuestionario de motivaciones, actitudes y comportamientos en el ocio f sico-deportivo. Logro o: Universidad de la Rioja.

29. Romero, G., \& Amador R. (2008). H bitos fisico-deportivos de universitarios colombianos. Bogot : Kinesis.

30. Rosset, J., \& Odam, G. (2010). El cuerpo del m sico. Manual de mantenimiento para un $\mathrm{m}$ ximo rendimiento. Barcelona: Paidotribo.

31. Sanz, E. (2005). La pr ctica f sico-deportiva de tiempo libre en universitarios. Logro o: Universidad de la Rioja.

32. Sard, E. (2003). En forma: ejercicios para m sicos. Madrid: Paid s.

33. Schoeb, V., \& Zosso A. (2012). "You cannot perform music without taking care of your body": a qualitative study on musicians' representation of body and health. Medical Problems of Performing Artists, 27(3), 129-136.

34. Torres, A. (2015). Resultados de sedentarismo en la poblaci $\mathrm{n}$ de primer semestre de 2010 de la Universidad Santo Tom s. Hallazgos, 7(14), 149-158.

35. Trainor, S., Delfabbro, P., Anderson, S., \& Winefield, A. (2010). Leisure activities and aolescente psychological wll-being. Journal of Adolescence, 33, 173-186.

36. Tubiana, R., \& Amadio, P. (2000). Functional anatomy. In: R. Tubiana \& P. Amadio, Medical problems of the performing instrumentalist (pp.14). London: Martin Dunitz Ltda.

37. Via o, J. (2009). Trastornos musculo-esquel ticos relacionados con la interpretaci $\mathrm{n}$ musical en estudiantes instrumentistas. Epidemiolog a y factores de riesgo. Actividad $\mathrm{f}$ sica y deporte, $\mathrm{h}$ bitos de prevenci $\mathrm{n}$ y carga f sica. New York: Human Movement.

38. World Leisure and Recreation Association (1994). WLRA International charter for leisure education. World Leisure \& Recreation, 36(2), 13-16.

39. Zaza, C. (1998). Playing-related musculoskeletal disorders in musicans: a systematic review of incidence and prevalence. Canadian Medical Association Journal, 158(8), 158-189. 